\title{
Idiopathic Thrombocytopenic Purpura in Pregnancy
}

\author{
${ }^{1} \mathrm{D}$ Sithara, ${ }^{2} \mathrm{KS}$ Rajeswari, ${ }^{3} \mathrm{M}$ Sivasundari
}

\begin{abstract}
Idiopathic thrombocytopenic purpura (ITP) is a diagnosis of exclusion. It is an autoimmune disorder caused by development of IgG autoantibodies, directed against a number of platelet glycoproteins. A 26 years, primigravida, booked from 30 weeks of gestation, admitted at Sri Ramachandra Medical College and Hospital at 36 weeks of gestation, with gestational hypertension and severe thrombocytopenia with a platelet count of $45,000 / \mathrm{mm}^{3}$. She was treated with intravenous steroids during her antenatal period for thrombocytopenia. She delivered a healthy baby girl of weight $2.4 \mathrm{~kg}$ by cesarean section and was breastfed. Intraoperatively, platelet transfusion was given. Postoperatively, she was on methyl prednisolone following which a good increment in the platelet count was noticed and then discharged. The aim is to clarify when thrombocytopenia in pregnancy is clinically important, to provide guidance regarding diagnosis, management options and information about potential risks to the mother and the fetus along with the review of relevant literatures.
\end{abstract}

Keywords: Corticosteroid, Idiopathic thrombocytopenia, Pregnancy.

How to cite this article: Sithara D, Rajeswari KS, Sivasundari M. Idiopathic Thrombocytopenic Purpura in Pregnancy. J South Asian Feder Obst Gynae 2015;7(2):95-96.

Source of support: Nil

Conflict of interest: None

Date of received: 30 May 2015

Date of acceptance: 10 June 2015

Date of publication: August 2015

\section{INTRODUCTION}

Idiopathic thrombocytopenic purpura (ITP) is an autoimmune disorder, with a platelet count often $<80 \times 10^{9} / 1$, induced by platelet-specific IgG antibodies. ${ }^{3}$ The great concern of ITP during pregnancy is the risk of thrombocytopenia in the newborn infant. ${ }^{2}$ ITP accounts for 3 to $4 \%$

\footnotetext{
${ }^{1}$ Postgraduate, ${ }^{2}$ Professor, ${ }^{3}$ Associate Professor

1-3 Department of Obstetrics and Gynecology, Sri Ramachandra Medical College and Hospital, Chennai, Tamil Nadu, India
}

Corresponding Author: D Sithara, Postgraduate, Department of Obstetrics and Gynecology, Sri Ramachandra Medical College and Hospital, Porur, Chennai-600116, Tamil Nadu, India, e-mail: sdjoe389@gmail.com of the cases of thrombocytopenia detected in pregnancy. It has many common causes which includes gestational thrombocytopenia, bacterial and viral infections, preeclampsia complicated by HELLP syndrome. ${ }^{1}$ We present here a case of ITP in pregnancy for its rarity and unique presentation who posed a therapeutic challenge.

\section{CASE REPORT}

A 26-year-old primigravida who presented in her 30th week of gestation with severe epistaxis. Her platelet count was ranging between 10,000 and $30,000 / \mathrm{mm}^{3}$ for which she was treated with intravenous steroids. Steroids were also covered for prematurity. She was diagnosed to have dimorphic anemia and was on iron, folic acid and vitamin B12. Her ANA and anti-dsDNA were negative. At 36th week of gestation, she had intermittent headache and was admitted for safe confinement. Admission platelet count was $45,000 / \mathrm{mm}^{3}$ and, hence, patient was started on intravenous steroids again, where the platelet counts increased to $1.051 / \mathrm{mm}^{3}$. At the end of $37 \mathrm{th}$ week of gestation, she had increased blood pressure and complaints of intermittent headache. She was started on labetalol and nifedipine. Magnetic resonance imaging of brain was done to rule out intracranial bleeding which reported a normal study. Hematology opinion was obtained and advised intravenous steroids. Blood bank intimated in prior for the provision of blood and blood products and single donor platelets. On the day of the 38th week of gestation, she had imminent signs of eclampsia and was started on parenteral magnesium sulphate and levetiracetam. Thus, she was admitted at 36 weeks of gestation for gestational hypertension along with ITP, who was followed in the hospital and was decided for termination of pregnancy at 38 weeks of gestation in view of imminent signs of eclampsia. After getting high-risk consent, patient underwent emergency lower segment cesarean section. Intraoperatively, she was transfused with 4 units of platelet concentrates and liquor was found to be thick meconium stained. She delivered a baby girl of weight $2.4 \mathrm{~kg}$ with a good Apgar score of $8 / 10$ and 9/10. Baby was seen by the neonatologist and was found healthy and breastfeeding was established successfully. Postoperatively, she was started on high dose methyl prednisolone. The platelet 
counts improved to $1.81 / \mathrm{mm}^{3}$ on the ninth postoperative day and she was discharged home.

\section{DISCUSSION}

Idiopathic thrombocytopenic purpura affects 1 to 3 per 1000 pregnancies. ${ }^{4}$ During pregnancy, hemodilution caused by relative increase in plasma volume coupled with increased platelet turnover leads to the development of thrombocytopenia, accounting for three quarters of cases detected during pregnancy. Idiopathic thrombocytopenic purpura is of three degrees. Mild thrombocytopenia, platelet count $<1.5 \mathrm{l} / \mathrm{mm} .{ }^{3}$ Moderate thrombocytopenia, platelet count $<11 / \mathrm{mm}$. ${ }^{3}$ Severe thrombocytopenia, platelet count $<0.5 \mathrm{l} / \mathrm{mm}$. ${ }^{3}$ Presenting symptoms include bruising, epistaxis, gum bleeding, petechial rash, more significant hemorrhage, however, increasingly asymptomatic women are diagnosed. Diagnostic approach is the same as in the non-pregnant state. Exclusion of all other causes of thrombocytopenia and other possible autoimmune disorders, and exclusion of $\mathrm{HIV}$ is mandatory. Effects on pregnancy include affection on the fetus and of the mother. Intracranial hemorrhage, the most feared complication of neonatal thrombocytopenia. Maternal issues include spontaneous bleeding posing a low incidence. If planning for delivery, platelet requirements include $>50,000 / \mathrm{mm}^{3}$ for vaginal delivery, $>80,000 / \mathrm{mm}^{3}$ for emergency cesarean section and usually 80,000 to $1,00,000 / \mathrm{mm}^{3}$ for elective cesarean section. What constitutes a safe platelet level for pregnancy and delivery has not been determined. Treatment options are just the same as in the nonpregnant women. First line is with corticosteroids, especially prednisone, which is metabolized by the placenta, so has minimal fetal side effects but increases the risk of coexisting conditions, like gestational diabetes, hypertension infections, preterm labor for the mother. Splenectomy can be considered if not responding to medical therapy. Laproscopic approach can be safely carried out in the second trimester. Intravenous immunoglobulin, swamps the IgG Fc receptors of macrophages in spleen, providing platelet count improvements. Rituximab, a monoclonal antibody therapy, is not clear though, as it crosses the placenta causing temporary suppression of B lymphocytes and long-term effect on infant's immune system development. Thrombocytopenia may limit the choices of anesthesia, but the mode of delivery is determined by obstetric indications. Finally, all neonates born to ITP mothers should be screened. Treatment options include intravenous immunoglobulins and platelet transfusion. In case of severe manifestations, rare possibility of coexistent neonatal autoimmune thrombocytopenia (NAIT) has to be excluded. ${ }^{3}$ Thus, it poses a diagnostic and a therapeutic challenge.

\section{REFERENCES}

1. Akther R, Hossain T, Khan MA et al. Pregnancy with idiopathic thrombocytopenic purpura-a case report. J Bangladesh Coll Phys Surg 2010;28(3):196-198.

2. Burrows RF, Kelton J. Pregnanacy in patients with idiopathic thrombocytopenic purpura: assssing the risks for the infant at delivery Obstet Gynecol Surv 1993 Dec;48(12):781-788.

3. Pourie, Raymond O, Greena, Michael F, Camann W. de Swiet's medical disorders in obstetric practice. Singapore: Wiley Blackwell; 2010. p. 808.

4. Gabbe, Steven G, Niebel, Jennifer R, Simpson, Joe L, et al. Textbook of obstetrics-normal and abnormal pregnancies. Philadelphia: Elsevier Health Sciences; 2012. p. 1272. 